\title{
On weighting the rates in non-response weights
}

\author{
Roderick J. Little ${ }^{1, *, \dagger}$ and Sonya Vartivarian ${ }^{2}$ \\ ${ }^{1}$ Department of Biostatistics, University of Michigan, 1420 Washington Heights, Ann Arbor, \\ MI 48109-2029, U.S.A. \\ ${ }^{2}$ Department of Statistics, University of Michigan, Ann Arbor, U.S.A.
}

\begin{abstract}
SUMMARY
A basic estimation strategy in sample surveys is to weight units inversely proportional to the probability of selection and response. Response weights in this method are usually estimated by the inverse of the sample-weighted response rate in an adjustment cell, that is, the ratio of the sum of the sampling weights of respondents in a cell to the sum of the sampling weights for respondents and non-respondents in that cell. We show by simulations that weighting the response rates by the sampling weights to adjust for design variables is either incorrect or unnecessary. It is incorrect, in the sense of yielding biased estimates of population quantities, if the design variables are related to survey non-response; it is unnecessary if the design variables are unrelated to survey non-response. The correct approach is to model non-response as a function of the adjustment cell and design variables, and to estimate the response weight as the inverse of the estimated response probability from this model. This approach can be implemented by creating adjustment cells that include design variables in the cross-classification, if the number of cells created in this way is not too large. Otherwise, response propensity weighting can be applied. Copyright (C) 2003 John Wiley \& Sons, Ltd.
\end{abstract}

KEY WORDS: sampling weights; survey inference; unit non-response adjustment

\section{INTRODUCTION}

Weighting is the standard method of non-response adjustment for surveys subject to unit nonresponse, where entire interviews are missing due to non-contact or refusal to answer the questionnaire. Respondents and non-respondents are classified into adjustment cells based on covariate information recorded for both groups, and respondents in cell $c$ are weighted by the inverse of the response rate in cell $c$. The sampling weight for each respondent is then multiplied by this non-response weight to obtain a combined weight for subsequent analysis.

Weighting for non-response is a natural extension of weighting for sample selection. The sampling weight (say $\pi_{i}^{-1}$ ) of a sampled unit $i$ is the inverse of the probability of selection,

\footnotetext{
* Correspondence to: Roderick Little, Department of Biostatistics, University of Michigan, 1420 Washington Heights, Ann Arbor, MI 48109-2029, U.S.A.

† E-mail: rlittle@umich.edu
}

Contract/grant sponsor: CDC; contract/grant number: UR6/CCU517481.

Copyright (c) 2003 John Wiley \& Sons, Ltd. 
and can be interpreted as the number of units in the population that unit $i$ is 'representing'. In particular suppose $y_{i}$ is the value of a survey variable $Y$, and $T$ is the population total of $Y$. In the absence of non-response a natural estimator of $T$ is the Horvitz-Thompson (HT) estimator [1] $\sum \pi_{i}^{-1} y_{i}$, where the sum is over sampled units. The HT estimator is an unbiased estimator of $T$ with respect to the randomization distribution. Although it can have unacceptably high variance [2], it is a useful all-purpose estimator in large samples.

In the presence of non-response, let $\pi_{i}^{-1}$ be the sampling weight and $w_{i}$ the non-response weight for responding unit $i$. The product $\pi_{i}^{-1} w_{i}$ can be interpreted as the number of units in the population represented by unit $i$. An obvious extension of the HT estimator of $T$ is then

$$
\hat{T}=\sum \pi_{i}^{-1} w_{i} y_{i}
$$

where the sum is over units that are sampled and respond. This estimate is approximately design unbiased for $T$, provided the respondents in cell $c$ are a random subsample of the sampled units in cell $c$. Since covariate information for unit non-response adjustments is often limited, this proviso is often a hope rather than an expectation. However, it is often plausible that unit non-response adjustment at least reduces the bias.

Note that $w_{i}$ is a sample quantity estimated from data, unlike the sample weight $\pi_{i}^{-1}$ which is determined by the sample design. This paper concerns the form of $w_{i}$ for unequal probability samples, more precisely for samples where the sampling weights are not constant within the adjustment cells. Suppose respondent $i$ falls in adjustment cell $c$. A naive choice is $w_{i}=1 / \hat{\phi}_{c}$, where $\hat{\phi}_{c}$ is the unweighted response rate

$$
\hat{\phi}_{c}=r_{c+} / n_{c+}
$$

and $n_{c+}$ and $r_{c+}$ are the number of sampled and responding individuals in cell $c$. If the sample weights are not constant within cell $c, \hat{\phi}_{c}$ is not an unbiased estimate of the population response rate in cell $c$ (that is, the proportion of the population that would respond if sampled). An (at least approximately) unbiased estimate of this quantity is the weighted response rate

$$
\hat{\phi}_{c}=\sum_{k \in R_{c}} \pi_{k}^{-1} / \sum_{k \in S_{c}} \pi_{k}^{-1}
$$

where $S_{c}$ and $R_{c}$ denote the set of units in cell $c$ that are sampled, and the set that are sampled and respond, respectively. One might compute the non-response weight $w_{i}$ in (1) as the inverse of the weighted rate in (3).

Should response rates be weighted, as in equation (3), or not weighted, as in equation (2)? Platek and Gray [3] discuss both methods, but draw no conclusions about which is to be preferred in practice. In a review of Census Bureau adjustment procedures, Chapman et al. [4] state that 'non-response adjustment factors are usually either the inverse of the survey's unweighted non-response rate, or an analogous ratio based on weighted survey counts'. Practice appears to favour weighted response rates. A recent enquiry to the list server for the Survey Research Methods Section of the American Statistical Association suggests that weighted response rates (3) are routinely used by the major survey research organizations. For example, the survey design for the National Health Interview Survey [5] oversamples Black and Hispanic households relative to other of races within secondary sampling units (SSUs), and then computes weighted response weights (3) within adjustment cells consisting of SSUs. To 
judge from their description, the non-response weights for the National Crime Survey appear to be unweighted, but cross-sectional weighting adjustments for the Survey of Income and Program Participation are currently weighted [6].

We argue in this paper that neither of these approaches is correct. The correct approach is to use the inverse of the unweighted rate (2), for adjustment cells that condition on both covariate and design information. In essence, the argument is that: (a) adjustment cells should be created to be homogeneous with respect to the propensity to respond; (b) if adjustment cells are created in this way, then weighting the non-response rates is unnecessary and inefficient, that is, it adds variance to estimates; and (c) if adjustment cells are created that are not homogeneous with respect to the propensity to respond, then weighting the response rates does not yield unbiased estimates of the means of population outcomes, even though the weighted response rates are unbiased estimates of the population response rates within each adjustment cell. Given (c), the right approach is not to weight the non-response rates, but rather to create adjustment cells based on a classification of the observed variables and the survey design variables, to control for association between survey stratifiers and non-response. Section 2 provides simulations in support of these statements. On the other hand, joint stratification on the adjustment cell variable and $Z$, the survey design variables, may achieve reduced nonresponse bias at the expense of increased variance, if the resulting adjustment cells are too sparse; approaches to that problem are discussed in Section 3.

\section{SIMULATION STUDY}

A simulation study was conducted to provide more insights into the variance and bias of estimators (2), (3) and alternatives, under a variety of population structures and non-response mechanisms. Categorical variables were simulated to avoid the need for distributional assumptions such as normality.

\subsection{Description of the population}

A population of size $N=10000$ was generated on a binary stratifier $Z$, observed for all units of the population, a binary adjustment cell variable $X$ observed for the sample, and a binary survey outcome $Y$ observed only for unit respondents. Also let $S$ denote the sampling indicator, observed for all units in the population, and $R$ the response indicator, observed for all units in the sample. The joint distribution of these variables, say $[Z, X, Y, S, R]$, can be factorized as follows:

$$
[Z, X, Y, S, R]=[Z, X][Y \mid Z, X][S \mid Z, X, Y][R \mid Z, X, Y, S]
$$

The distributions on the right side are then defined as follows:

(i) Distribution of $Z$ and $X$. The joint distribution of $[Z, X]$ was multinomial, with $\operatorname{pr}(Z=X=0)=0.3, \operatorname{pr}(Z=0, X=1)=0.4, \operatorname{pr}(Z=1, X=0)=0.2$ and $\operatorname{pr}(Z=X=1)=$ 0.1 , yielding the population counts in Table I.

(ii) Distribution of $Y$ given $X, Z$. Values of the survey variable $Y$ were generated according to the logistic model

$$
\operatorname{logit} P(Y=1 \mid X, Z)=0.5+\gamma_{X}(X-\bar{X})+\gamma_{Z}(Z-\bar{Z})+\gamma_{X Z}(X-\bar{X})(Z-\bar{Z})
$$


Table I. Population counts of $X$ and $Z$.

\begin{tabular}{lrr}
\hline & $Z=0$ & $Z=1$ \\
\hline$X=0$ & 3064 & 2079 \\
$X=1$ & 3931 & 926 \\
\hline
\end{tabular}

Table II. Models for $Y$ given $X, Z$.

\begin{tabular}{lccc}
\hline Model & $\gamma_{X}$ & $\gamma_{Z}$ & $\gamma_{X Z}$ \\
\hline 1. $[X Z]^{Y}$ & 2 & 2 & 2 \\
2. $[X+Z]^{Y}$ & 2 & 2 & 0 \\
3. $[X]^{Y}$ & 2 & 0 & 0 \\
4. $[Z]^{Y}$ & 0 & 2 & 0 \\
5. $[\phi]^{Y}$ & 0 & 0 & 0 \\
\hline
\end{tabular}

for five choices of $\gamma=\left(\gamma_{X}, \gamma_{Z}, \gamma_{X Z}\right)$ chosen to reflect different relationships between $Y$ and $X$ and $Z$. These choices are displayed in Table II, using conventional generalized linear model notation. Here the additive logistic model is labelled $[X+Z]^{Y}$, and sets the interaction $\gamma_{X Z}$ to zero, whereas the model $[X Z]^{Y}$ sets this interaction equal to 2. Models where $Y$ depend on $X$ only, $Z$ only or neither $X$ nor $Z$ are denoted by $[X]^{Y},[Z]^{Y}$ and $[\phi]^{Y}$, respectively.

(iii) Distribution of $S$ given $Z, X$ and $Y$. The sample cases were assumed to be selected from the population using stratified random sampling, so $S$ is independent of $X$ and $Y$ given $Z$, that is $[S \mid Z, X, Y]=[S \mid Z]$. The probabilities of selection were $\pi_{0}=262 / 6995$ (about 0.04) when $Z=0$ and $\pi_{1}=50 / 3005$ (about 0.02) when $Z=1$.

(iv) Distribution of $R$ given $Z, X, Y$ and $S$. Since the response mechanism is assumed ignorable and the selection is by stratified random sampling, $[R \mid Z, X, Y, S]=[R \mid Z, X]$. The latter is generated by a logistic model

$$
\operatorname{logit} P(R=1 \mid X, Z)=0.5+\beta_{X}(X-\bar{X})+\beta_{Z}(Z-\bar{Z})+\beta_{X Z}(X-\bar{X})(Z-\bar{Z})
$$

where $\beta=\left(\beta_{X}, \beta_{Z}, \beta_{X Z}\right)$ takes the same values found in Table II, with $\gamma$ replaced by $\beta$. We also ran the simulation with a negative interaction term, but the results were similar. As for the distribution of $Y$ given $X$ and $Z$, this yields five models for the distribution of $R$ given $X$ and $Z$. For example, $[X+Z]^{R}$ refers to $R$ being additively dependent on $X$ and $Z$.

There were thus a total of $5 \times 5=25$ combinations of population structures and non-response mechanisms in the simulation study. A total of 1000 replicate data sets were generated for each of the 25 combinations. Table III displays the form of nine estimators, computed for each data set, of the overall mean $\bar{Y}=N^{-1} \sum_{j} \sum_{k} N_{j k} \bar{Y}_{j k}$, where $N_{j k}$ and $\bar{Y}_{j k}$ are the number of population units and the population mean, respectively, with $Z=j, X=k$. The first estimator is the weighted response rate estimator (3) based on adjustment cells $X$, labelled $\operatorname{wrr}(x)$, and the second estimator is the analogous unweighted response estimator (2), labelled $\operatorname{urr}(x)$. 
WEIGHTING THE RATES IN NON-RESPONSE WEIGHTS

Table III. Estimators of mean of $Y$.

\begin{tabular}{|c|c|c|c|c|}
\hline \multicolumn{2}{|c|}{ Response weight Assumed model } & Estimator & Weight & Response rate \\
\hline 1. $\operatorname{wrr}(x)$ & & $\frac{\sum_{x} \sum_{z} w_{x z}^{*} r_{x z} \bar{y}_{x z}}{\sum_{x} \sum_{z} w_{x z}^{*} r_{x z}}$ & $w_{x z}^{*}=\frac{\left(N_{+z} / n_{+z}\right)(r / N)}{\hat{\phi}_{x}^{*}}$ & $\hat{\phi}_{x}^{*}=\frac{\sum_{z}\left(r_{x z} / \pi_{z}\right)}{\sum_{z}\left(n_{x z} / \pi_{z}\right)}$ \\
\hline 2. $\operatorname{urr}(x)$ & & $\frac{\sum_{x} \sum_{z} w_{x z} r_{x z} \bar{y}_{x z}}{\sum_{x} \sum_{z} w_{x z} r_{x z}}$ & $w_{x z}=\frac{\left(N_{+z} / n_{+z}\right)(r / N)}{\hat{\phi}_{x}}$ & $\hat{\phi}_{x}=\frac{r_{x+}}{n_{x+}}$ \\
\hline 3. $\operatorname{ml}(x z) / \operatorname{urr}(x z)$ & {$[X Z]^{Y}$} & $\frac{\sum_{x} \sum_{z} w_{x z}^{\prime} r_{x z} \bar{y}_{x z}}{\sum_{x} \sum_{z} w_{x z}^{\prime} r_{x z}}$ & $w_{x z}^{\prime}=\frac{\left(N_{+z} / n_{+z}\right)(r / N)}{\hat{\phi}_{x z}}$ & $\hat{\phi}_{x z}=\frac{r_{x z}}{n_{x z}}$ \\
\hline 4. $\mathrm{ml}(x)$ & {$[X]^{Y}$} & $\frac{\sum_{x} w_{x}^{*} \bar{y}_{x}}{\sum_{x} w_{x}^{*}}$ & $w_{x}^{*}=\sum_{z} w_{x z}^{\prime} r_{x z}$ & \\
\hline 5. $\mathrm{ml}(z)$ & {$[Z]^{Y}$} & $\sum_{z} \frac{N_{z}}{N} \bar{y}_{z}$ & & \\
\hline 6. $\mathrm{ml}($ null $)$ & {$[\phi]^{Y}$} & $\sum_{z} \frac{r_{z}}{r} \bar{y}_{z}$ & $w_{x}^{*}=\sum_{z} w_{x z}^{\prime} r_{x z}$ & \\
\hline 7. $\mathrm{ml}(x+z)$ & {$[X+Z]^{Y}$} & $\frac{\sum_{x} \sum_{z} w_{x z}^{\prime} r_{x z}\left(\hat{\mu}+\hat{\alpha}_{1 x}+\hat{\alpha}_{2 z}\right)}{\sum_{x} \sum_{z} w_{x z}^{\prime} r_{x z}}$ & & \\
\hline 8. $\operatorname{urr}(x+z)$ & {$[X Z]^{Y}$} & $\frac{\sum_{x} \sum_{z} w_{x z}^{(u)} r_{x z} \bar{y}_{x z}}{\sum_{x} \sum_{z} w_{x z}^{(u)} r_{x z}}$ & $w_{x z}^{(u)}=\frac{\left(N_{+z} / n_{+z}\right)(r / N)}{\hat{\phi}_{x z}^{(u)}}$ & $\begin{array}{l}\hat{\phi}_{x z}^{(u)} \text { from unweighted } \\
\text { additive logistic model }\end{array}$ \\
\hline 9. $\operatorname{wrr}(x+z)$ & {$[X Z]^{Y}$} & $\frac{\sum_{x} \sum_{z} w_{x z}^{(w)} r_{x z} \bar{y}_{x z}}{\sum_{x} \sum_{z} w_{x z}^{(w)} r_{x z}}$ & $w_{x z}^{(w)}=\frac{\left(N_{+z} / n_{+z}\right)(r / N)}{\hat{\phi}_{x z}^{(w)}}$ & $\begin{array}{c}\hat{\phi}_{x z}^{(w)} \text { from weighted } \\
\text { additive logistic model }\end{array}$ \\
\hline
\end{tabular}

The next five estimators are maximum likelihood (ML) for the assumed models relating $Y$ to $X$ and $Z$ listed in the second column of Table III. These estimates all have the form $\hat{Y}=\sum_{j} \sum_{k} \hat{P}_{j k} \hat{Y}_{j k}$, where $\hat{P}_{j k}=\left(N_{j+} / N\right)\left(n_{j k} / n_{j+}\right)$ is the ML estimate of the proportion of the population with $Z=j, X=k$, and:

1. If the model for $Y$ is $[X Z]^{Y}$, then $\hat{Y}_{j k}=\bar{y}_{j k}$.

2. If the model for $Y$ is $[X+Z]^{Y}$, then $\hat{Y}_{j k}=\hat{\mu}+\hat{\alpha}_{1 j}+\hat{\alpha}_{2 k}$, predicted values from an additive logistic model fitted to the respondent data.

3. If the model for $Y$ is $[X]^{Y}$, then $\hat{Y}_{j k}=\bar{y}_{+k}$.

4. If the model for $Y$ is $[Z]^{Y}$, then $\hat{Y}_{j k}=\bar{y}_{j+}$.

5. If the model for $Y$ is $[\phi]^{Y}$, then $\hat{Y}_{j k}=\bar{y}_{++}$.

It is interesting to note that neither of the weighting class estimators $\operatorname{urr}(x)$ and $\operatorname{wrr}(x)$ are ML for any of the models used to generate the data in this simulation study. On the other hand, the estimator that weights by the response rates in cells based on the classification by $Z$ and $X$ is ML for the saturated model $[X Z]^{Y}$; this estimator is denoted as $\operatorname{urr}(x z)$ in Table III. The last two estimators in Table III, $\operatorname{wrr}(x+z)$ and $\operatorname{urr}(x+z)$, both obtain the estimate the mean of $Y$ in cell $j k$ as $\hat{Y}_{j k}=\bar{y}_{j k}$. These estimators involve response rates that are predictions from an additive logistic model for $R$ on $X$ and $Z$, where for $\operatorname{urr}(x+z)$ the cases in the logistic regression are weighted equally, and for $\operatorname{wrr}(x+z)$ the cases are 
weighted by the inverse of the probability of selection. These methods are closely related to the response propensity stratification discussed in Section 3.

Table IV shows the average root mean square error (RMSE) of the nine estimators in Table III over the 1000 replicate data sets, a measure that takes into account both precision and bias. Asymptotic properties of ML lead us to believe that the ML estimator for a particular assumed model will have close to the lowest RMSE when the assumed model is the same as the model used to generate the data. Table $\mathrm{V}$ displays the average bias over the 1000 replicates, defined to be the average of the difference of the estimator before deletion of cases due to non-response and the estimator based on respondents alone.

Table VI shows for selected pairwise comparisons whether differences in performance between the estimates are statistically significant. The table displays

$$
\bar{d}=(1 / 1000) \sum_{i=1}^{1000} d_{i}, \quad \text { where } d_{i}=\left|\theta_{B D i}-\hat{\theta}_{1 i}\right|-\left|\theta_{B D i}-\hat{\theta}_{2 i}\right|
$$

$\theta_{B D i}$ is the mean before deletion of cases due to non-response for the $i$ th replicate, and $\hat{\theta}_{1 i}$ and $\hat{\theta}_{2 i}$ are pairs of estimates of the mean of $Y$ as found in the first row of Table VI. A negative value indicates the first estimator $\hat{\theta}_{1 i}$ does better than $\hat{\theta}_{2 i}$, whereas a positive value indicates $\hat{\theta}_{2 i}$ does better. The standard error of $\bar{d}$ is computed as the standard deviation of the individual $d_{i}$ 's divided by $\sqrt{ } 1000$, and differences that are statistically significant from zero based on a $t$-test are asterisked $\left({ }^{*}=P<0.05,{ }^{* *}=P<0.01\right)$.

A crude summary of the relative performance of the methods is the RMSE averaged over all problems, shown in the last row of Table IV. Note that the best methods all stratify on both $X$ and $Z$, and have similar average RMSE:

$$
\operatorname{urr}(x z)=382, \quad \operatorname{ml}(x+z)=380, \quad \operatorname{wrr}(x+z)=383, \quad \operatorname{urr}(x+z)=381
$$

The methods that stratify on $X$ but not $Z$ are much worse than these methods in overall RMSE:

$$
\operatorname{urr}(x)=471, \quad \operatorname{wrr}(x)=471, \quad \operatorname{ml}(x)=443
$$

with the slightly better performance of $\mathrm{ml}(x)$ reflecting gains in efficiency when the model is true. The methods that stratify on $Z$ but not $X$ are worst of all in overall RMSE:

$$
\operatorname{ml}(z)=507, \quad \operatorname{ml}(\text { null })=528
$$

although as expected these methods show some gains of efficiency in populations where $Y$ does not depend on $X$.

As expected, the ML estimate for the model used to generate the data is always best or close to best in these simulations. The estimate for the additive model $[X+Z]^{Y}$ is theoretically biased when the data-generating model includes the $X Z$ interaction, but in these simulations the bias for the overall mean of $Y$ is modest.

The unweighted response weight estimator $\operatorname{urr}(x)$ is biased and performs poorly when both $Y$ and $R$ depend on $Z$, since in these cases the stratification on $Z$ cannot be ignored. Note, however, that weighting the response weights, as in $\operatorname{wrr}(x)$, does not generally correct the bias of $\operatorname{urr}(x)$ in these situations: $\operatorname{wrr}(x)$ performs very similarly to $\operatorname{urr}(x)$, and in fact as we have seen its average RMSE over all problems is the same. Two interesting cases where $\operatorname{wrr}(x)$ 
WEIGHTING THE RATES IN NON-RESPONSE WEIGHTS

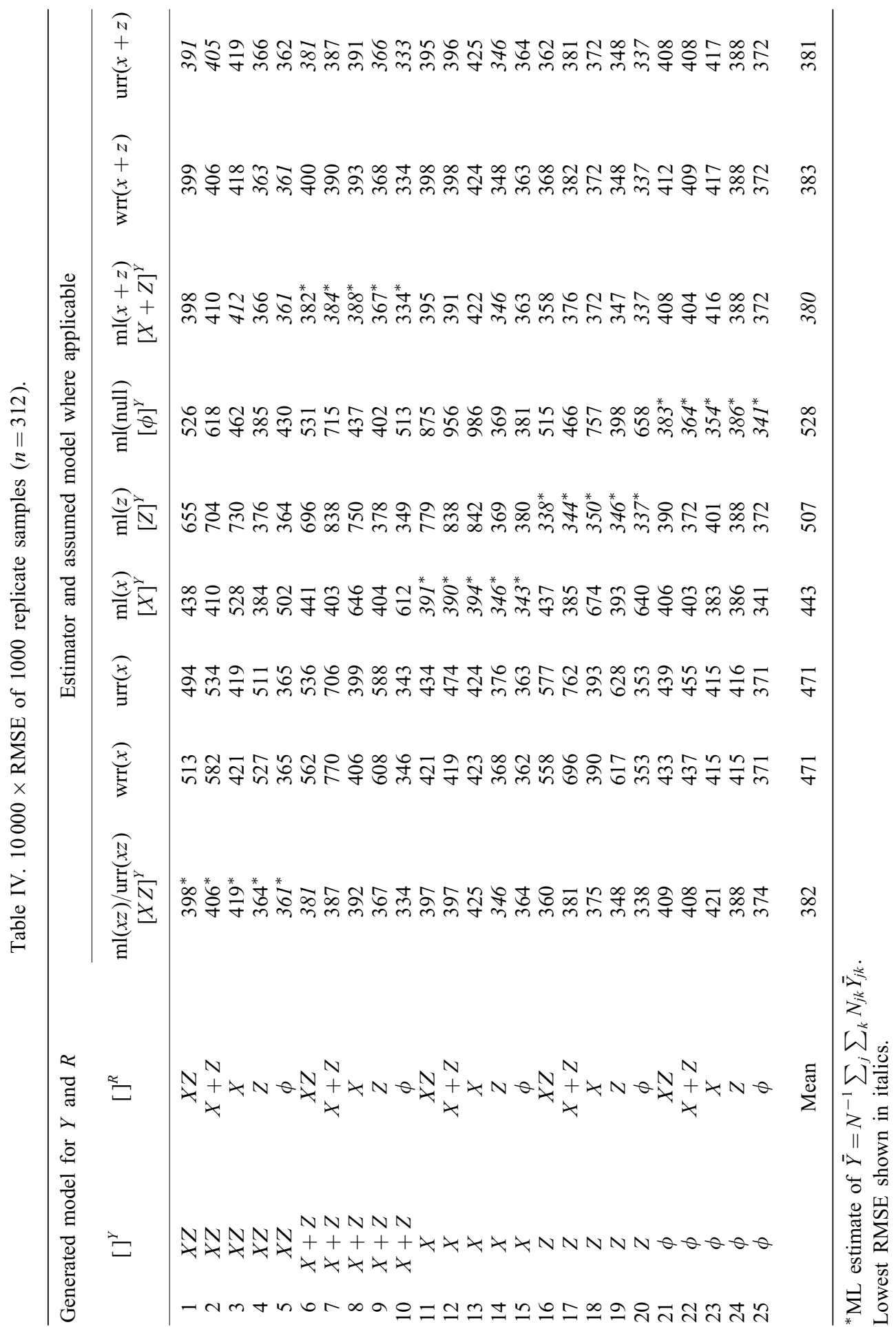




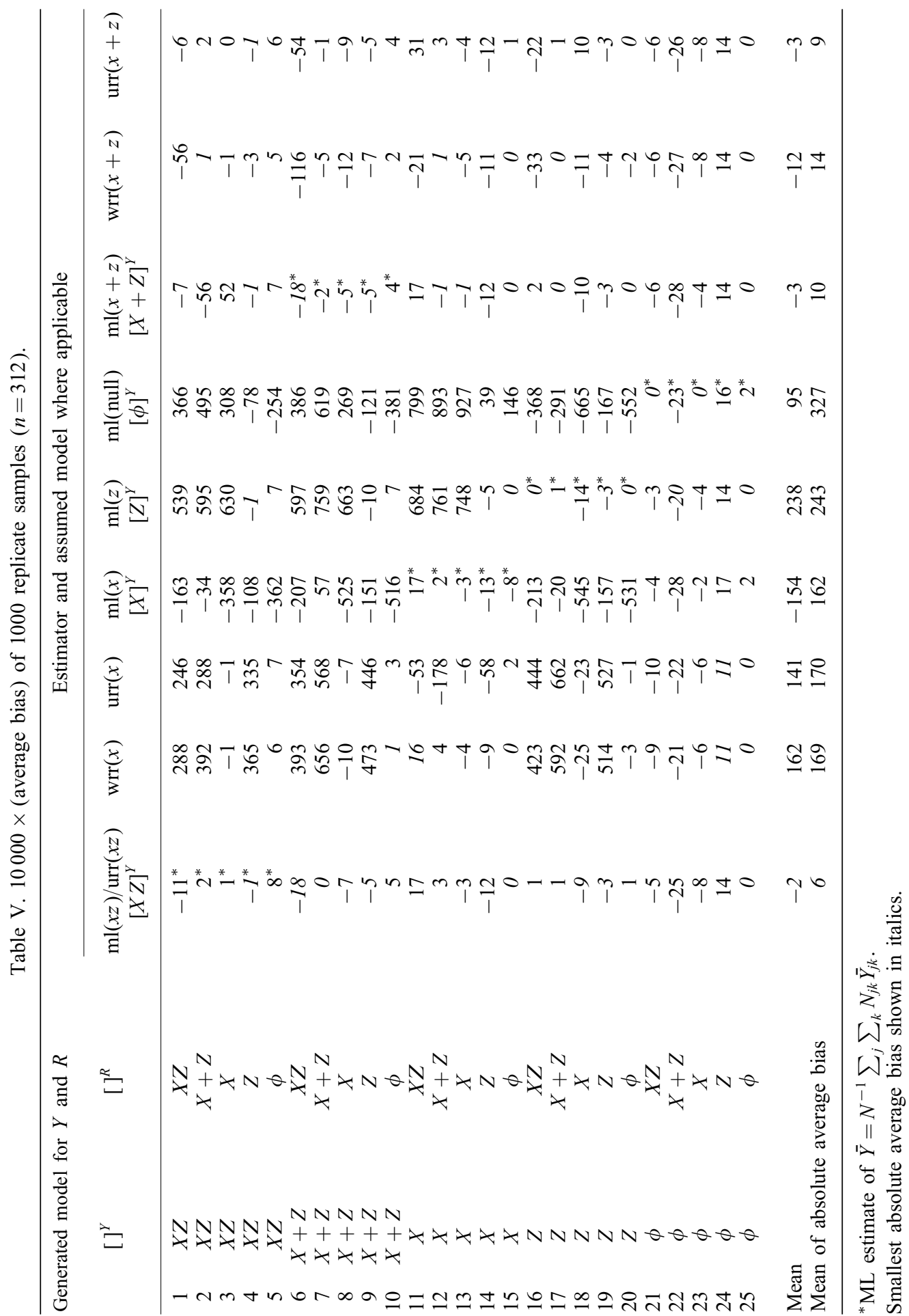


WEIGHTING THE RATES IN NON-RESPONSE WEIGHTS

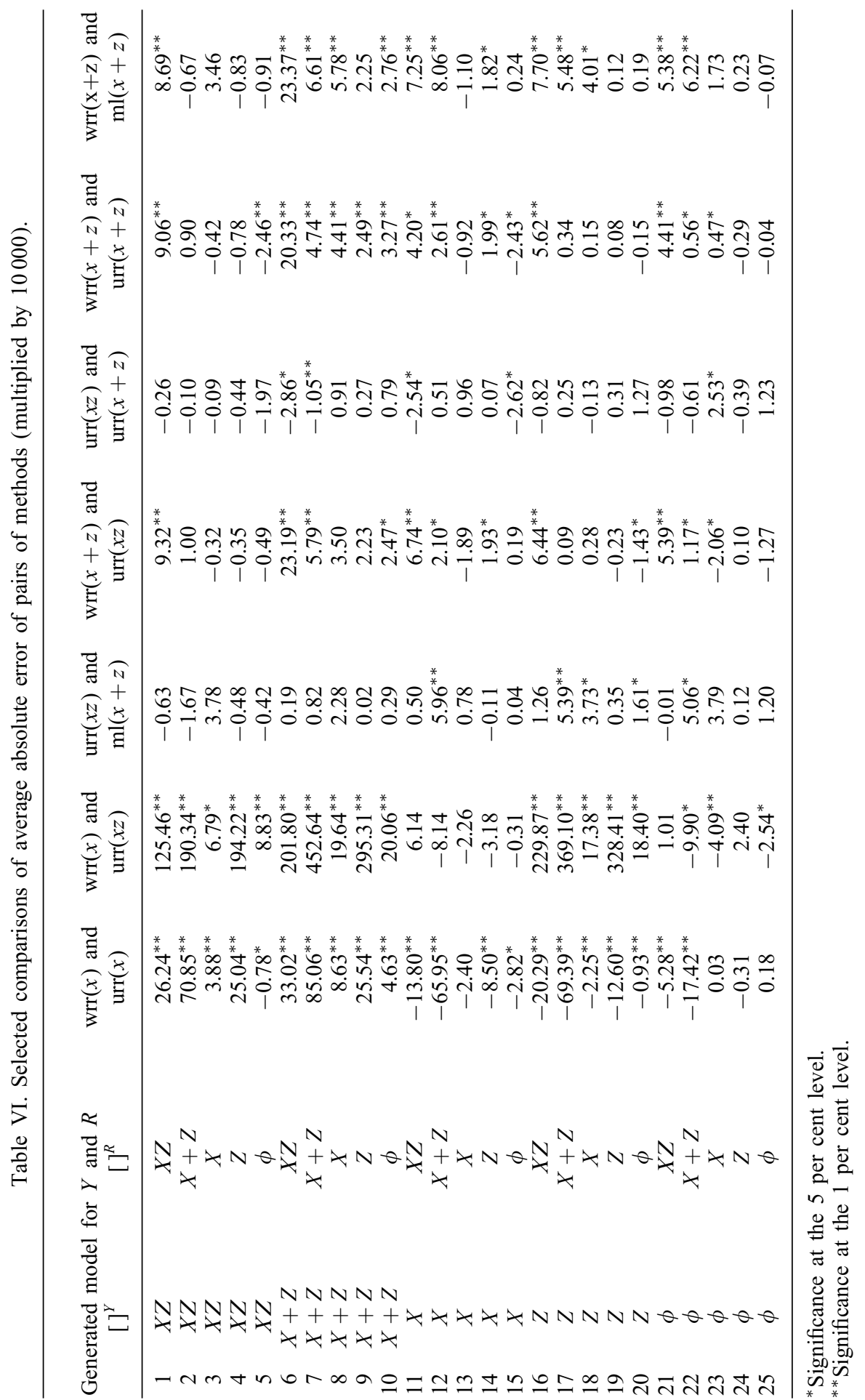


does improve on $\operatorname{urr}(x)$ are where $R$ depends on both $X$ and $Z$ and $Y$ depends on $X$ but not $Z$ (specifically the models $[X]^{Y},[X Z]^{R}$ and $[X]^{Y},[X+Z]^{R}$ ), in rows 11 and 12 of the tables). In these cases, weighting the response rates yields unbiased response rate estimates in the cells defined by $X$, and the respondent mean of $Y$ in these cells is unbiased since $Y$ depends only on $X$. However, the gain in weighting the response rates in these cases is relatively minor, and (as might be predicted) $\mathrm{ml}(x)$ is superior to either method in these cases. Also, the practical importance of these cases is debatable: $Y$ is likely to depend on $Z$ as well as $X$, since the point of stratifying on $Z$ is to exploit the relationship between $Y$ and $Z$. The estimator $\operatorname{urr}(x z)$ that stratifies on both $X$ and $Z$ is robust under all of the models, and does much better overall than either $\operatorname{urr}(x)$ or $\operatorname{wrr}(x)$.

The estimators that base the estimated response rates on an additive logistic model, namely $\operatorname{wrr}(x+z)$ and $\operatorname{urr}(x+z)$, perform well, though neither are ML for any of the generating models. Unlike $\operatorname{wrr}(x)$ and $\operatorname{urr}(x), \operatorname{wrr}(x+z)$ and $\operatorname{urr}(x+z)$ both take the design variable into account by obtaining separate estimates of the response rate for cells that stratify both on $X$ and $Z$. Their performance is similar to $\operatorname{ml}(x+z)$ and $\operatorname{urr}(x z)$, with $\operatorname{wrr}(x+z)$ doing slightly worse overall. Weighting the logistic regressions does not appear to offer any advantage here.

\section{GENERAL STRATEGIES FOR CREATING ADJUSTMENT CELLS}

For the relatively simple situations simulated in Section 2, with just two strata and two values of $X$, adjustment cells can be created based on the joint distribution of $Z$ and $X$. In more realistic settings, the cross-classification of the survey design variables and observed survey variables can yield too many adjustment cells, some of which may contain sampled cases but no respondents. For example, in the Health Interview Survey [5], weighted response weights are calculated within the second-stage sampling unit (SSU), a variable that has many levels. Joint classification by $Z$ and $X$ would correspond to stratifying households within the SSU according to race, which would yield many small adjustment cells, including perhaps some with no respondents. Thus a strategy is needed for reducing the number of adjustment cells. Two such strategies are discussed in this section.

Let $D$ denote the complete set of variables recorded for both respondents and nonrespondents, including design variables and any survey variables measured for both groups. (For unit non-response survey variables are usually entirely absent for non-respondents, but in panel surveys variables from earlier surveys may be available.) We say that non-response is ignorable if the distribution of the incomplete survey variables is the same for respondents and non-respondents with the same value of $D$. Formally, if $R$ is an indicator for response or non-response, $Y$ is the set of survey variables missing for non-respondents, then non-response is ignorable if

$$
R \coprod Y \mid D
$$

where $\amalg$ denotes independence. Adjustments for non-ignorable non-response are usually highly speculative, and all the methods discussed in this paper effectively assume that non-response is ignorable. Thus we assume that (6) holds.

We have noted that adjustment cells defined by each distinct value of $D$ may be too small and yield weights that are undefined or too unstable. Thus the problem becomes to define adjustment cells based on $D$ that remove non-response bias, whilst avoiding sparse 
cells that lead to unstable weights, and resulting estimates with large variance. Two sensible objectives in defining adjustment cells are (a) to choose cells that are homogeneous with respect to outcome variables $Y$, and (b) to choose cells that are homogeneous with respect to the probability of response. Theory supporting both these choices is presented in Little [7], who considers two methods for creating adjustment cells when $D$ is extensive: (i) predictive mean stratification, motivated by objective (a), groups units according to predicted means of $Y$ given $D$, estimated for example by regression of $Y$ on $D$ based on the responding cases; (ii) response propensity stratification, motivated by objective (b), groups units according to their estimated probabilities of response, computed for example by logistic regression of the response indicator $R$ on $D$ based on sampled cases. Little [7] showed that if $\hat{Y}(D)$ denotes the predicted mean of $Y$ given $D$, and $\hat{p}(D)$ denotes the predicted probability of response given $D$, then (with some additional conditions described in the paper), (6) implies that

$$
Y \coprod R \mid \hat{Y}(D)
$$

and

$$
Y \coprod R \mid \hat{p}(D)
$$

In particular assuming ignorable non-response and ignoring the effects of estimating $\hat{Y}(D)$ and $\hat{p}(D)$, weighting based on either of these methods of stratification removes non-response bias in estimating population means. Of these two methods, only response propensity stratification also removes bias of estimates of means for subclasses of the population [7]. This theory supports the idea of basing weights based on a model for the propensity to respond on $D$, where the latter includes the design variables that determine the sampling weight. This approach is closely related to the $\operatorname{urr}(x+z)$ method in the simulation study, which was competitive with the best methods. Weighting the logistic regression by the sampling weight, as in $\operatorname{wrr}(x+z)$, did not offer any advantage in our simulations, and by analogy with the simpler case of stratification on $x$ alone, we do not expect any advantages of weighting in more complex situations.

\section{ACKNOWLEDGEMENTS}

This research was supported by CDC grant UR6/CCU517481. We appreciate helpful comments from Michael Brick, David Judkins and Trivellore Raghunathan.

\section{REFERENCES}

1. Horvitz DG, Thompson DJ. A generalization of sampling without replacement from a finite universe. Journal of the American Statistical Association 1952; 47:663-685.

2. Basu D. An essay on the logical foundations of survey sampling, Part 1. In Foundations of Statistical Inference. Holt, Rinehart and Winston: Toronto, 1971; 203-242.

3. Platek R, Gray GB. Imputation methodology. In Incomplete Data in Sample Surveys, Vol. 2: Theory and Bibliographies, Madow WG, Olkin I, Rubin DB (eds). Academic Press: New York, 1983; 255-294.

4. Chapman DW, Bailey L, Kasprzyk D. Nonresponse adjustment procedures at the U.S. Bureau of the Census. Survey Methodology 1986; 12:161-180.

5. Botman SL, Moore TF, Moriarty CL, Parsons VL. Design and estimation of the National Health Interview Survey, 1995-2004. National Center for Health Statistics, Vital Health Statistics 2000; 2:130.

6. Petroni R, King KE. Evaluation of Survey of Income and Program Participation's cross-sectional noninterview adjustment. Proceedings of the Survey Research Methods Section, American Statistical Association 1988; 342-347.

7. Little RJ. Survey nonresponse adjustments. International Statistical Review 1986; 54:139-157. 